\title{
Erythrocyte cell membrane phospholipid levels compared against reported dietary intakes of polyunsaturated fatty acids in pregnant Mexican women
}

\author{
Maria-Socorro Parra ${ }^{1, *}$, Lourdes Schnaas ${ }^{2}$, Mohsen Meydani ${ }^{3}$, Estela Perroni ${ }^{2}$, \\ Sandra Martínez ${ }^{2}$ and Isabelle Romieu ${ }^{1}$ \\ ${ }^{1}$ National Institute of Public Health Mexico, Ave. Universidad 655, Col. Sta. Ma. Ahuacatitlán, Cuernavaca, \\ Morelos, Mexico 62508: ${ }^{2}$ Developmental Neurobiology Department, National Institute of Perinatology, Mexico \\ City, Mexico: ${ }^{3}$ Jean Mayer USDA Human Nutrition Research Center on Aging at Tufts University, Boston, MA, USA
}

\begin{abstract}
Objective: To evaluate the validity of a food-frequency questionnaire (FFQ) for assessment of the dietary intakes of polyunsaturated fatty acids (PUFAs) against a biochemical marker of fat intake, erythrocyte cell membrane phospholipid levels, during pregnancy.

Design: Cross-sectional analysis.

Setting: Developmental Neurobiology Department, National Institute of Perinatology, Mexico City.

Subjects: One hundred forty-six healthy pregnant women during the last trimester of pregnancy. Among women enrolled, the first 35 pregnant women (24\%) had their erythrocytes analysed for fatty acid status.

Methods: We administered an FFQ and compared intakes of PUFAs against their erythrocyte cell membrane concentrations, processed by gas chromatography.

Results: Pearson correlation coefficients among $\alpha$-linolenic acid (ALN), docosahexaenoic acid (DHA) and eicosapentaenoic acid in erythrocyte cell membranes against their crude dietary counterparts were $0.32,0.35$ and 0.36 (each $P<0.05$ ). In a simple linear regression, erythrocyte DHA and arachidonic acid (AA) were significantly related to their respective dietary intakes $(\beta=0.30,95 \%$ confidence interval (CI): $0.007-0.60, P=0.045$ for DHA; $\beta=0.49,95 \%$ CI: $0.010-0.98, P=0.044$ for AA). Erythrocyte cell membrane ALN concentration (\%/total) was only marginally related to ALN dietary intake $\left(\mathrm{mg} \mathrm{day}^{-1}\right)(\beta=0.52,95 \% \mathrm{CI}:-0.020-1.10, P=0.061)$ However, after adjustment for long-chain $n-3$ PUFA/AA, this association reached significance ( $\beta=0.44,95 \%$ CI: $0.026-0.825, P=0.038$ ). Main dietary sources for $n-3$ PUFAs were canned tuna fish and fresh catfish; for $n-6$ these were eggs and cow's milk. The use of this FFQ in these pregnant Mexican women provided estimates of average long-term intakes of PUFAs and correlated reasonably well with their erythrocyte cell membrane phospholipid status. However, we need to consider that, during pregnancy, there is a faster turnover of PUFAs from fat storage that may modify the profile of erythrocyte PUFAs and lower the correlation between dietary intake and erythrocyte PUFAs.
\end{abstract}

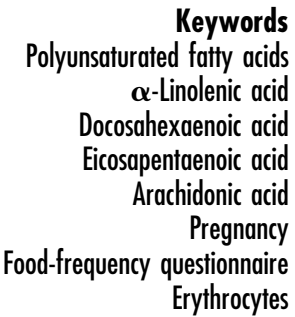

Polyunsaturated fatty acids (PUFAs) and especially their metabolic derivatives, the long-chain PUFAs (LCPUFAs), are structural components of cell membrane phospholipids, affecting membrane signalling and many other vital functions within the perinatal period ${ }^{1-4}$. These fatty acids are essential to humans, because they cannot be synthesised and need to be ingested by food intake ${ }^{5}$. Owing to the roles PUFAs play as functional modulators, it has been stated that maternal, foetal and neonatal LCPUFA status are important determinants of health and disease later in life $\mathrm{e}^{6,7}$

During the last three months of pregnancy, there is a rapid accumulation of PUFAs in foetal brain tissue. A recent workshop in the USA, attended by experts in the field, acknowledged the need to monitor the diets of pregnant and lactating women to verify whether they are adequate in long-chain $n-3$ fatty acids, given the structural role played by docosahexaenoic acid (DHA) in the brain and retina, and the rapid brain development that takes place during the last trimester of pregnancy and infancy ${ }^{1-7}$. In particular, a specific focus of scientific interest is the balance between intakes of the two families of PUFAs ( $n-3$ and $n-6$ ), because they interact metabolically, and change in the ratio between them may influence the kind of eicosanoids that will be formed ${ }^{1,2}$.

Dietary intake reports remain a matter of debate 
because many inaccuracies occur when recalling food habits $^{8,9}$. Biochemical validation makes it possible to compare food reports collected by dietary assessment methods against biochemical markers ${ }^{10,11}$. In the case of these fatty acid compounds, good correlations have been documented between dietary intake of eicosapentaenoic acid (EPA) and eicosanoid levels in the cell membranes of erythrocytes in non-pregnant ${ }^{11-13}$ and pregnant subjects $^{2,14-17}$.

This paper evaluates the associations between the intakes of PUFAs, obtained through a food-frequency questionnaire (FFQ), and the relative amounts in erythrocytes.

\section{Materials and methods}

This study was part of a larger study undertaken to investigate the suggested relationship between PUFAs ingested during pregnancy and foetal neurodevelopment. The study took place from August 1999 until September 2000. Pregnant women who were screened for inclusion in the study were among those scheduled to attend a routine third-trimester prenatal visit at the prenatal clinic at the National Institute of Perinatology in Mexico City. Exclusion criteria were: younger than 18 years of age or older than 42 years; having active diabetes, hepatitis or fat metabolism disease, as determined by laboratory test; habitual use of alcohol or drugs; taking prescription medications; high blood pressure; and active psychosis. Eligible women who agreed to participate signed an informed consent. An FFQ validated for use in Mexican women was administered by a trained interviewer ${ }^{18}$. We also administered a questionnaire to gather socioeconomic and demographic variables, and information on drug and tobacco use, as well as reproductive history. A venous blood sample was taken, processed and stored during this first visit. The Ethics Committee at the National Institute of Public Health of Mexico approved the research protocol.

\section{Study subjects}

Among eligible women, 97.3\% (146 out of 150) were enrolled. Among them, the first 35 pregnant women (24\%) had their erythrocytes analysed for fatty acid status. Mean weight gain during the last two months of pregnancy was $4.2 \mathrm{~kg}$ (standard deviation (SD) $1.5 \mathrm{~kg}$ ). Thirty-six per cent of the women were younger than 25 years and $28.1 \%$ were older than 34 years of age.

\section{Dietary intake}

We used a semi-quantitative FFQ including 104 items. This questionnaire has already been validated in Mexican women residing in Mexico City ${ }^{18}$. In brief, it included 10 food sections and an additional section inquiring about the dietary sources of $n-3$ PUFAs: fresh fish, sardines, tuna and seafood (average of $1 / 2$ cup containing shrimp, crab, shellfish and octopus). We developed a new version of the FFQ by adding extra food items as sources for $n-3$ PUFAs such as cod oil, fresh and canned salmon, and typical dry fish found in central Mexico, called charales. The questionnaire already had a section on oils to account for the $n-6$ fatty acids (including safflower oil, olive oil, soya oil and canola oil); a section on other meat sources for arachidonic acid (AA) such as beef and pork; and a section on vegetable sources such as green leafy products. The questionnaire was administered to the mother by a trained and standardised nutritionist who inquired about the average frequency of consumption of each food item during the past year. The frequencies ranged from never up to 6 times per day (never, less than once per month, 13 times per month, 1-2 per week, 3-4 per week, 5-6 per week, daily, 2-3 times per day, $4-5$ times per day and 6 or more per day). The weights used to account for frequency were the proportional ones to transform all reported frequencies into once per day: never $=0$, once per month $=0.016,1-3$ times per month $=0.08$, once per week $=0.14,2-4$ times per week $=0.43,5-6$ times per week $=0.8$, once per day $=1.0,2-3$ times per day $=2.5,4-5$ times per day $=4.5$ and 6 times per day $=6$. There was only one possible choice to answer per foodstuff. The fruit and vegetable sections took account of seasonal availability ${ }^{18}$.

\section{Calculation of nutrient intakes}

Although there are two institutions working on determining the PUFA contents of foodstuffs in Mexico, to date there is no available food composition table that includes the contents of PUFAs and/or their metabolic derivatives in Mexican foods. Therefore, we had to rely on the US Department of Agriculture's (USDA) food composition tables available online through the Internet ${ }^{19}$, although we are aware that the PUFA content in fish consumed in Mexico might be slightly different. Inconsistencies in composition may also derive from the different analytical techniques used to determine these nutrients ${ }^{8}$. The estimated contents of the various food items used to quantify total intake of $n-3$ and $n-6$ fatty acids are provided in Table 1 . We calculated the grams per day of specific PUFAs that were consumed as an average in this population, using the previously mentioned weights. Calculations were performed using a special software called SNUT 3.0 (nutriment system), developed at the National Institute of Public Health, Mexico, for this purpose and patented at the Ministry of Education, Copyright Division in 1999. Energy-adjusted dietary $n-3$ and $n-6$ fatty acids were calculated by the regression method proposed by Willett ${ }^{7}$. We also calculated the percentage of adequacy between the dietary intakes of these participants and the Recommended Dietary Allowances (RDA) from the US National Research Council ${ }^{20}$. 
Table 1 Food concentrations ( $\mathrm{g}$ ) of fatty acids according to USDA values and Mexican FFQ portion sizes. Also grams consumed per day according to frequency of consumption

\begin{tabular}{|c|c|c|c|c|c|c|c|c|c|}
\hline Food & $\begin{array}{l}\text { Portion } \\
\text { size }(g)\end{array}$ & $\begin{array}{l}\text { Consumption } \\
\left(\text { g day }^{-1}\right)\end{array}$ & $16: 1 n-7$ & $18: 1 n-9$ & $18: 2 n-6$ & $18: 3 n-3$ & $20: 4 n-6$ & $20: 5 n-3$ & $22: 6 n-3$ \\
\hline Eggs & 60 & 88.64 & 0.1788 & 2.0844 & 0.6888 & 0.0204 & 0.852 & 0.0024 & 0.0216 \\
\hline Tuna fish & 115 & 17.96 & 0.1525 & 1.9362 & 3.1331 & 0.5045 & 0.0587 & 0.2581 & 0.6806 \\
\hline Liver & 70 & 15.59 & 0.0345 & 0.4190 & 0.3154 & 0 & 0.2021 & 0 & 0.1750 \\
\hline Catfish & 130 & 11.75 & 0.2223 & 6.9511 & 3.4008 & 0.2314 & 0.0390 & 0.1547 & 0.2886 \\
\hline Sardine & 115 & 2.49 & 0.2530 & 2.4667 & 4.0744 & 0.5727 & 0 & 0.5439 & 0.5853 \\
\hline Seafood & 75 & 5.88 & 0.1102 & 0.0495 & 0.0755 & 0.0007 & 0.2452 & 0.0788 & 0.0758 \\
\hline Spinach & 30 & 31.95 & 0.0006 & 0.0006 & 0.0046 & 0.0253 & 0 & 0 & 0 \\
\hline Safflower & 10 & 126.43 & 0.0400 & 1.1688 & 7.4084 & 0.0399 & 0 & 0 & 0 \\
\hline Margarine & 4 & 33.75 & 0 & 0.0280 & 0.0240 & 0 & 0 & 0 & 0 \\
\hline Vegetable lard & 4 & 1.28 & 0.1240 & 1.6360 & 0 & 0.0440 & 0 & 0 & 0 \\
\hline Lamb & 130 & 9.06 & & & 1.63 & & & & \\
\hline Pork lard & 4 & 60.18 & 0.1079 & 9.0893 & 0.4074 & 0.0400 & 0 & 0 & 0 \\
\hline Whole milk & 200 & 88.64 & 0.1475 & 0.7213 & 0.1476 & 0.0983 & 0 & 0 & 0 \\
\hline
\end{tabular}

USDA - US Department of Agriculture; FFQ - food-frequency questionnaire.

\section{Erythrocyte fatty acid measurements}

The blood samples were chilled immediately in an ice bath, and erythrocytes were obtained within an hour by centrifuging the mothers' venous blood samples at $4{ }^{\circ} \mathrm{C}$. When isolated, the erythrocytes were washed three times with a saline solution (0.89\%). After this procedure, erythrocytes were put into cryotubes and stored in an ultra freezer at $-70^{\circ} \mathrm{C}$. Analyses were carried out at the Human Nutrition Research Center (HNRC) at Tufts University. After osmotic haemolysis, the erythrocyte cell membranes were washed at high-speed centrifugation. Lipids were extracted from erythrocyte cell membranes with chloroform-methanol $(2: 1 \mathrm{vol} / \mathrm{vol})$ and $\mathrm{KCl}(0.88 \%)$ in the presence of the antioxidant, butylated hydroxytoluene. Fatty acid methyl esters were prepared by acidcatalysed transmethylation with methanolic hydrochloride and injected into a gas chromatograph (Hewlett Packard 5890, Avondale, PA). The chromatograph was equipped with a flame ionisation detector and a $30 \mathrm{~m}$ fused silica capillary column, using a temperature programme rising from $130^{\circ} \mathrm{C}$ to $230^{\circ} \mathrm{C}$ by increments of $3^{\circ} \mathrm{C} \mathrm{min}{ }^{-1}$. Peaks were identified with the Chemstation (Hewlett Packard, 1997) chromatography program. Separated peaks from the detector output were identified by authentic fatty acids and expressed as weight percentage. Erythrocytes for fatty acid analyses were stored for no longer than 6 months. Within this period there was no sign of degradation of fatty acids. The coefficients of variation were $14: 0=6.7 \%$, $16: 0=1.8 \%, 16: 1=6 \%, 18: 0=2.5 \%, 18: 1 n-9=2.7 \%$, $18: 2 n-6=2.3 \%, \quad 18: 3 n-3=25.8 \%, \quad 20: 1 n-9=15.7 \%$, $20: 2 n-6=7.9 \%, \quad 20: 3 n-6=13.3 \%, \quad 20: 4 n-6=9.2 \%$, $20: 5 n-3=1.6 \%, \quad 22: 4 n-6=24 \%, \quad 22: 5 n-3=20.1 \%$, $22: 6 n-3=13.4 \%$, total fatty acids $=10.6 \%$.

The present paper focuses on determining the estimated intakes of PUFAs from the maternal diet during the last trimester of pregnancy through an FFQ, and on presenting the erythrocyte cell membrane PUFA status in a subsample of pregnant mothers during the third trimester of pregnancy, a relevant exposure period to these fatty acids for the foetus.

\section{Statistical metbods}

We estimated Pearson correlation coefficients between the crude and log-transformed dietary PUFA intakes and their biochemical concentrations. Simple linear regression models were used to evaluate the association between the dietary and the erythrocyte cell membrane PUFAs, crude and energy-adjusted. Finally, we determined the multiple linear regression coefficients between the dietary and biochemical PUFAs, adjusting for the $n-3$ LCPUFA/AA ratio ((EPA + DHA)/AA). We constructed quartiles according to the distribution in the study population for each dietary PUFA and cross-tabulated these data with their respective erythrocyte biomarkers to verify how well the FFQ worked at categorising future mothers.

\section{Results}

Mean intake of all kinds of fish was $32.2 \mathrm{~g} \mathrm{day}^{-1}$ and of oils was $393.5 \mathrm{mlday}^{-1}$ (Table 1). As expected, energyadjusted dietary $n-3$ and $n-6$ fatty acids tended to exhibit smaller standard deviations than did crude daily intakes (data not shown). Main dietary sources for $n-3$ PUFAs were canned tuna fish and fresh catfish; for $n-6$ these were eggs and cow's milk. In Table 2 , we present the dietary intakes of fatty acids $\left(\mathrm{mgday}^{-1}\right)$. The largest ranges of intakes were for fatty acids 16:0, 18:1, cis18:1n9 , and both parent carbons from $n-6$ and $n-3$ families: 18:2n-6 (linolenic acid, LA) and 18:3n-3 ( $\alpha$-linoleic acid, LNA). Pregnant women's erythrocyte status showed that the most abundant PUFAs were oleic acid (18:1n-9) and LA (18:2n-6). The ratio between the $n-6$ and $n-3$ families was approximately 2 (Table 3). Pearson correlation coefficients among $\alpha$-linolenic acid (ALN), DHA and EPA in erythrocytes against their own crude dietary concentrations were $0.32,0.35$ and 0.36 , respectively (each 
Table 2 Dietary fatty acid levels $\left(\mathrm{mg} \mathrm{day}^{-1}\right)$ obtained through the Mexican FFQ

\begin{tabular}{lrrrr}
\hline & $n$ & Mean \pm SD & Minimum & Maximum \\
\hline $4: 0$ & 146 & $0.583 \pm 0.27$ & 0.06 & 1.44 \\
$6: 0$ & 146 & $0.353 \pm 0.17$ & 0.04 & 0.85 \\
$8: 0$ & 146 & $0.204 \pm 0.10$ & 0.03 & 0.52 \\
$10: 0$ & 146 & $0.505 \pm 0.22$ & 0.07 & 1.20 \\
$12: 0$ & 146 & $0.617 \pm 0.28$ & 0.07 & 1.65 \\
$14: 0$ & 146 & $2.452 \pm 0.94$ & 0.36 & 5.42 \\
$16: 0$ & 146 & $14.989 \pm 5.12$ & 3.35 & 33.71 \\
$18: 0$ & 146 & $6.576 \pm 2.45$ & 1.16 & 15.84 \\
$16: 1$ & 146 & $1.697 \pm 0.62$ & 0.35 & 4.64 \\
$18: 1$ & 146 & $26.403 \pm 10.09$ & 5.58 & 64.96 \\
cis $18: 1 n-9$ & 146 & $17.895 \pm 7.56$ & 3.56 & 49.46 \\
trans18:1n-9 & 146 & $1.435 \pm 0.85$ & 0.10 & 7.85 \\
$20: 1$ & 146 & $0.137 \pm 0.07$ & 0.02 & 0.41 \\
$22: 1$ & 146 & $0.022 \pm 0.03$ & 0.00 & 0.13 \\
cis $18: 2 n-9$ & 146 & $10.394 \pm 5.03$ & 2.82 & 32.30 \\
trans18:2n-9 & 146 & $0.158 \pm 0.09$ & 0.00 & 0.88 \\
cis/trans18:2n-9 & 146 & $0.320 \pm 0.25$ & 0.00 & 2.30 \\
$18: 2 n-6$ & 146 & $14.795 \pm 8.92$ & 4.05 & 43.04 \\
$20: 4 n-6$ & 146 & $0.170 \pm 0.08$ & 0.01 & 0.39 \\
$22: 5 n-6$ & 146 & $0.005 \pm 0.01$ & 0.00 & 0.03 \\
$18: 3 n-3$ & 146 & $1.518 \pm 0.71$ & 0.30 & 5.86 \\
$18: 4 n-3$ & 146 & $0.007 \pm 0.01$ & 0.00 & 0.06 \\
$20: 5 n-3$ & 146 & $0.052 \pm 0.04$ & 0.00 & 0.26 \\
$22: 6 n-3$ & 146 & $0.140 \pm 0.11$ & 0.00 & 0.63 \\
\hline
\end{tabular}

FFQ - food-frequency questionnaire; SD - standard deviation.

$P \leq 0.05)$. After adjustment for total caloric intake, these correlations remained similar.

Mean energy intake was $2898.62 \mathrm{kcal}$ (SD, $770.67 \mathrm{kcal}$ ) of which approximately $31 \%$ was derived from fat, $56 \%$ from carbohydrate and 13\% from protein (Table 4). Animal fat accounted for $52.4 \%$ of total fat, probably due to the large consumption of pork lard. PUFAs accounted for up

Table 3 Erythrocyte cell membrane fatty acid concentrations (\%/total) of pregnant women

\begin{tabular}{lrrrr}
\hline & $n$ & \multicolumn{1}{c}{ Mean \pm SD } & Minimum & Maximum \\
\hline $14: 0$ & 35 & $0.5288 \pm 0.173$ & 0.250 & 0.940 \\
$16: 0$ & 35 & $21.3815 \pm 2.490$ & 12.915 & 24.874 \\
$16: 1 n-7$ & 35 & $0.9097 \pm 0.242$ & 0.586 & 1.459 \\
$18: 0$ & 35 & $16.1767 \pm 1.045$ & 13.935 & 18.389 \\
$18: 1 n-9$ & 35 & $17.8368 \pm 1.442$ & 14.054 & 21.628 \\
$20: 1 n-9$ & 35 & $0.9970 \pm 0.352$ & 0.543 & 1.874 \\
$n-9$ & 35 & $18.8338 \pm 1.730$ & 14.697 & 23.502 \\
$18: 2 n-6$ & 35 & $12.6096 \pm 1.268$ & 10.506 & 15.194 \\
$18: 3 n-6$ & 34 & $0.1634 \pm 0.076$ & 0.072 & 0.354 \\
$20: 2 n-6$ & 35 & $1.6270 \pm 0.620$ & 0.650 & 3.091 \\
$20: 3 n-6$ & 35 & $1.7542 \pm 0.557$ & 0.675 & 3.075 \\
$20: 4 n-6$ & 35 & $9.6901 \pm 2.299$ & 4.588 & 14.702 \\
$22: 4 n-6$ & 35 & $2.9532 \pm 0.756$ & 1.581 & 4.406 \\
$n-6$ & 34 & $28.6126 \pm 2.914$ & 22.831 & 34.210 \\
$18: 3 n-3$ & 35 & $1.8512 \pm 1.068$ & 0.297 & 4.458 \\
$20: 5 n-3$ & 35 & $1.3042 \pm 0.184$ & 0.991 & 1.820 \\
$20: 3$ & 34 & $0.3549 \pm 0.095$ & 0.146 & 0.577 \\
$22: 5 n-3$ & 35 & $3.7208 \pm 0.635$ & 1.066 & 4.465 \\
$22: 6 n-3$ & 35 & $6.0592 \pm 0.852$ & 4.842 & 8.085 \\
$n-3$ & 34 & $13.2586 \pm 1.420$ & 10.024 & 17.344 \\
Ratio $n-6 / n-3$ & 33 & $2.1767 \pm 0.351$ & 1.651 & 2.994 \\
$20: 4 n-6 / 22: 6 n-3$ & 35 & $1.6053 \pm 0.362$ & 0.841 & 2.377 \\
\hline & & & & \\
\hline $0-5 n d a r d ~$ & & & &
\end{tabular}

SD - standard deviation.

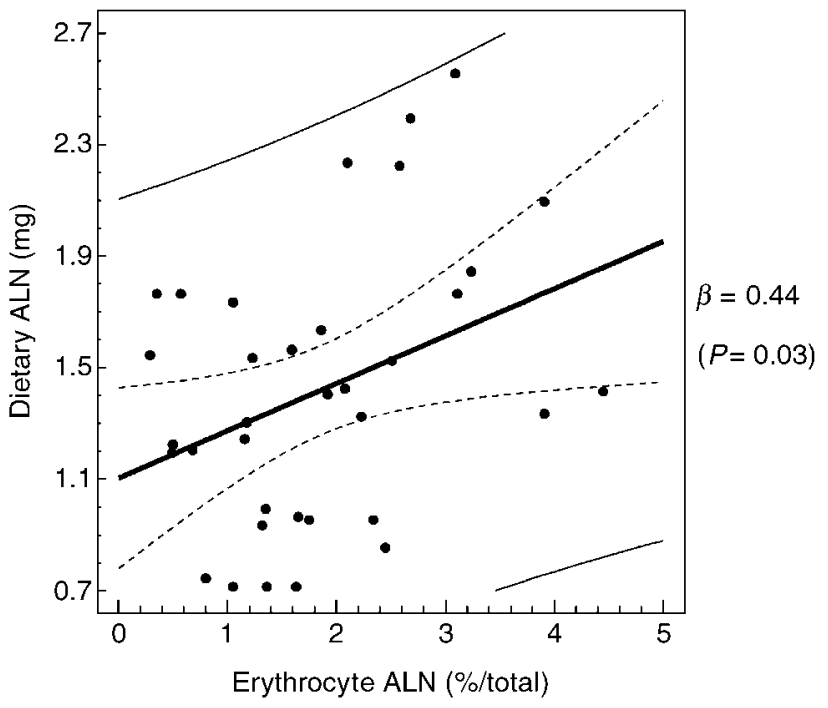

Fig. 1 Regression of erythrocyte cell membrane $\alpha$-linolenic acid (ALN; 18:3n-3) on dietary ALN, adjusted by the $n-3$ LCPUFA/AA ratio $((\mathrm{DHA}+\mathrm{EPA}) / \mathrm{AA})$. Thick black line - regression line; dotted lines $-95 \%$ confidence intervals for the regression line; thin black lines - outer segment

to $21 \%$ of total fat, and monounsaturated for $37 \%$. Antioxidants varied in their contribution to fulfil the RDA. Vitamin C intake corresponded to only $50 \%$ of the RDA, while vitamin E reached $133 \%$ of the RDA.

In linear regression models (Table 5), we observed that erythrocyte cell membrane PUFA was positively related to dietary intake for ALN (carbon parent of $n-3$ family) and the two main metabolic products from $n-3$ and $n-6$ families, DHA (22:6n-3) and AA (20:4n-6). These associations were statistically significant only for DHA and AA. However, when we adjusted by the ratio of $n-3$ LCPUFAs to AA, the association between erythrocyte ALN and ALN dietary intake reached statistical significance $(\beta=$ 0.44, 95\% CI: $0.026-0.825, P=0.038)$. Figure 1 shows a positive association between erythrocyte ALN (18:3n-3) and its adjusted dietary amount. Figure 2 shows the crude association between erythrocyte palmitoleic acid $(16: 1 n-7)$ and its dietary intake $(\beta=0.88,95 \% \mathrm{CI}$ : $0.11-0.86, P=0.024)$. In Fig. 3 , we present the crossclassification of the quartiles of erythrocyte ALN and ALN dietary intake. We observed that women classified in the lowest and highest quartiles of dietary intake were also classified in such quartiles by the erythrocyte ALN content.

\section{Discussion}

The present study documents that PUFA levels, measured in erythrocyte cell membrane phospholipids, reflect dietary intakes of the parent carbon of $n-3$ fatty acids and palmitoleic acid from the $n-7$ family in a group of pregnant Mexican women. Our FFQ is therefore adequate to identify the highest and lowest quartiles of intake among pregnant women according to $n-3$ fatty acid intakes. It needs to be stressed, however, that the 
Table 4 Energy and selected nutrient intakes obtained from FFQ in pregnant women

\begin{tabular}{lccrrr}
\hline Variable & $n$ & Mean \pm SD & Minimum & Maximum & \%RDA \\
\hline Energy (kcal) & 146 & $2898.62 \pm 770.67$ & 862.10 & 5165.61 & \\
Protein (g) & 146 & $92.72 \pm 23.72$ & 25.78 & 166.32 & \\
Carbohydrate $(\mathrm{g})$ & 146 & $414.71 \pm 121.40$ & 105.77 & 782.31 & \\
Total fat $(\mathrm{mg})$ & 146 & $103.43 \pm 31.99$ & 26.31 & 204.53 & \\
Animal fat $(\mathrm{mg})$ & 146 & $54.23 \pm 20.00$ & 9.84 & 139.56 & \\
Vegetable fat $(\mathrm{mg})$ & 146 & $49.20 \pm 17.97$ & 10.02 & 64.77 & \\
Monounsaturated $(\mathrm{mg})$ & 146 & $38.52 \pm 13.77$ & 6.90 & 87.41 & \\
Polyunsaturated $(\mathrm{mg})$ & 146 & $22.04 \pm 8.96$ & 5.31 & 50.70 & \\
Cholesterol $(\mathrm{mg})$ & 146 & $340.60 \pm 165.30$ & 51.32 & 884.72 & \\
Vitamin C $(\mathrm{mg})$ & 146 & $330.22 \pm 183.01$ & 63.06 & 1095.10 & 47.2 \\
Retinol $(\mu \mathrm{g})$ & 146 & $4924.29 \pm 3449.39$ & 691.92 & 15858.52 & \\
Vitamin $\mathrm{E}(\mathrm{mg} \alpha$-TE) & 146 & $13.26 \pm 5.25$ & 3.70 & 28.59 & 133.0 \\
\hline
\end{tabular}

FFQ - food-frequency questionnaire; SD - standard deviation; $\alpha$-TE- $\alpha$-tocopherol equivalents. ${ }^{\star}$ Recommended Dietary Allowance for pregnant women from US National Research Council ${ }^{20}$.

measurements were done in the third trimester of pregnancy in women with an uncomplicated pregnancy, and that results cannot be extrapolated freely beyond these selection criteria.

The categorisation obtained by comparing erythrocyte cell membrane phospholipid levels and intakes as measured by the FFQ worked reasonably well for the first and fourth quartiles, which are the most important comparison groups in an epidemiological study. However, the linear trend across quartiles was weak. Thus the method selected for collecting dietary data can be judged as valid and reflecting intakes of PUFAs. However, when using energy-adjusted figures we did not find an improvement of correlations, perhaps due to the fact that the concentrations of these PUFAs are quite small and that adjustment might not have a great impact on reducing variability, when using the residual method.

A correlation coefficient of 0.32 for ALN is relatively high compared with findings from other observational studies

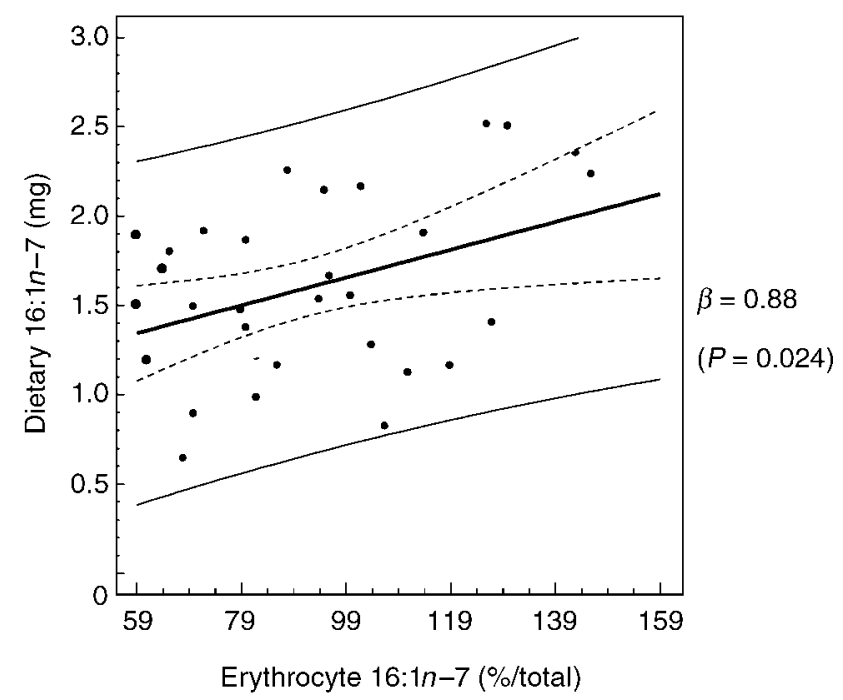

Fig. 2 Regression of erythrocyte cell membrane palmitoleic acid $(16: 1 n-7)$ on dietary 16:1n-7. Thick black line - regression line; dotted lines - $95 \%$ confidence intervals for the regression line; thin black lines - outer segment correlating biochemical markers to estimated dietary intakes. In a similar analysis, Olsen et $a l .{ }^{14}$ reported a correlation coefficient of 0.02 for ALN, probably because in their population dietary sources of ALN were lower than in our study and dietary intakes of DHA and EPA were proportionally higher. Our regression model between erythrocyte cell membrane ALN and ALN dietary intake improved when adjusting for the $n-3$ LCPUFA/AA ratio, which suggests that this ratio influences the metabolic pathway of PUFAs. Moreover, this ALN, the parent carbon from the $n-3$ family, was the only one to show good correlation with its biochemical counterpart. Olsen et al. ${ }^{14}$ reported that, in a group of 135 pregnant women in Denmark, the main sources of $n-3$ were mackerel, cod liver, cooked lean fish and cooked medium-fat fish. Hulshof et $a l^{21}$ found that, in women in 14 European countries, ALN intake ranged from $2.5 \mathrm{~g} \mathrm{day}^{-1}$ in Denmark

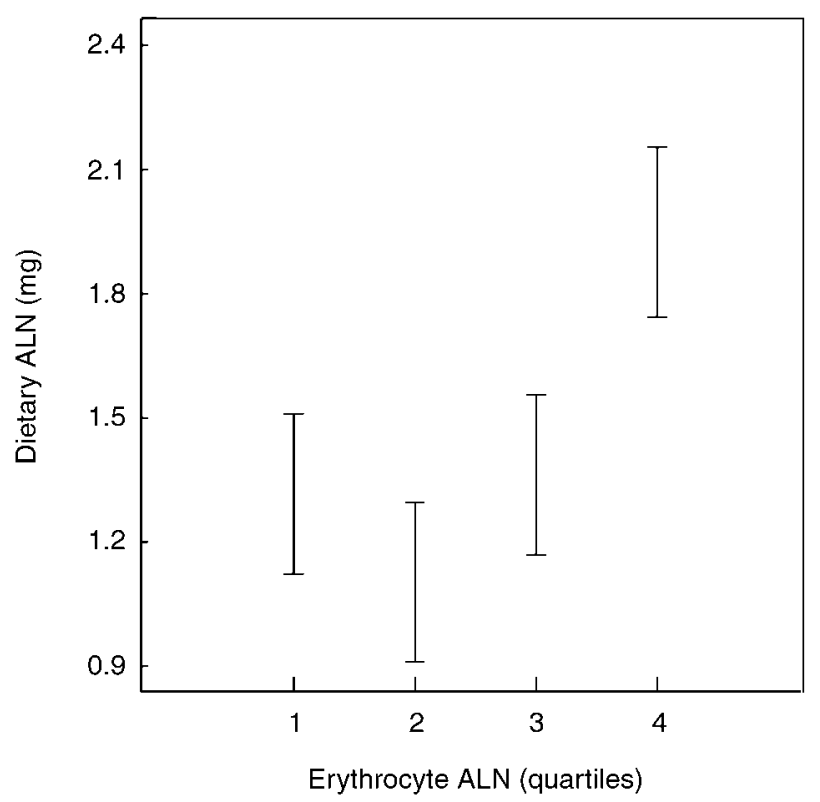

Fig. 3 Quartiles ( $1=$ lowest; $4=$ highest) according to population distribution of erythrocyte cell membrane $\alpha$-linolenic acid (ALN; $18: 3 n-3)$ concentrations tabulated against dietary consumption of ALN 
Table 5 Linear regression coefficients between erythrocyte cell membrane polyunsaturated fatty acid (PUFA) levels (\%/total) and log-transformed dietary PUFAs

\begin{tabular}{lccr}
\hline Dietary PUFAs & Coefficient & $95 \% \mathrm{Cl}$ & $P$-value \\
\hline$\alpha$-Linolenic acid (18:3n-3) & 0.52 & $-0.020-1.10$ & 0.061 \\
Docosahexaenoic acid (22:6n-3) & 0.30 & $0.007-0.60$ & 0.045 \\
Arachidonic acid (20:4n-6) & 0.49 & $0.010-0.98$ & 0.044
\end{tabular}

$\mathrm{Cl}$ - confidence interval.

(as the highest) to $0.6 \mathrm{~g} \mathrm{day}^{-1}$ in France, while in our population was the intake was $1.52 \mathrm{~g} \mathrm{day}^{-1}$. This places our population between Norway and Sweden (1.6 $\mathrm{g} \mathrm{tday}^{-1}$ and $1.4 \mathrm{~g} \mathrm{tday}^{-1}$, respectively). In our population this intake of ALN could have been due to the high intake of milk $\left(88.6 \mathrm{~g} \mathrm{day}^{-1}\right)$, which is a rich source of this fatty acid ${ }^{1}$.

Different studies have reported PUFA levels in erythrocytes. When compared with our results, Hornstra et $a .^{22}$ found a lower erythrocyte concentration of ALN ( $50 \%$ less), but a higher concentration of palmitoleic acid (54\% higher) in a group of 15 pregnant women from The Netherlands. However, our sum of $n-7$ and $n-9$ PUFAs was larger than theirs (19.74\% vs. $13.80 \%)$, which reflects the deficiency in $n-6$ and $n-3$ in our study population. In contrast, Araya and colleagues ${ }^{23}$ found a lower ratio of $n-7$ plus $n-9(12.0 \%)$ than in our population, in a group of 11 Chilean pregnant women. In addition, Chilean women had lower erythrocyte ALN concentration than observed in ours and Hornstra et al.'s study (4.3\%, 12.61\% and $21.7 \%$, respectively). Neither Hornstra et al.'s nor Araya et al.'s study reported dietary intake of the participants.

Several factors are likely to attenuate the associations. (1) The measurement errors inherent in the two methods; (2) incomplete knowledge to construct appropriate models for the time-effect and dose-response relationships between the intakes of $n-6$ and $n-3$ fatty acids and their appearance in erythrocyte phospholipids; and (3) body levels of $n-6$ as well as $n-3$ fatty acids may be influenced by factors other than the amounts consumed, e.g. interpersonal variation in the absorption and $\beta$ oxidation of $n-3$ fatty acids, and in the export of $n-3$ fatty acids to the foetus during the last trimester of pregnancy.

Using the FFQ questionnaire to rank individuals requires information on major but also discriminating dietary sources between individuals; however, there are only a limited number of questions that can be included. In our questionnaire we used eight food items related to $n-3$ PUFA intake, but averaged all types of fresh fish without clear distinction between oily and non-oily fish, except for salmon. This may have led to a misclassification of PUFA intakes. In addition, our questionnaire relies on predetermined portion sizes, which may also have led to measurement error. However, we believe that women did not consciously misreport their intake given that, in Mexico, most people are not aware of the potential benefits of consuming these products especially during the third trimester of pregnancy.

It should also be taken into account that although dietary assessment methods are acceptable to determine population dietary intakes, the so-called food intake assessment evaluations from remote past work are better for categorising individuals according to population distributions. These categories can then be useful to estimate epidemiological risks from dietary exposures and link them to health outcomes. However, this method relies on individual recall, which may be a source of measurement error. In addition, apart from variations in food composition, the method of food preparation may cause oxidation of highly unsaturated fatty acids and modify their content in foods. Von Houwelingen et al. ${ }^{24}$ stated that, to obtain an estimate of the possible metabolic interactions between the various fatty acids studied, we have to explore further the influence of dietary ALN on uptake and/or metabolism of EPA and DHA acids from the $n-3$ family in serum or erythrocytes, taking into account the dietary amounts.

Another important issue to discuss is the type of biochemical marker used. Arab and $\mathrm{Akbar}^{25}$ stated that biomarkers of PUFAs can be confounded by the fact that they reflect relative percentages and not absolute amounts. In addition, factors that influence fat deposition and mobilisation differ between and within individuals according to endogenous factors such as metabolic state (especially pregnant women) and synthesis, and exogenous factors such as PUFA intakes. The method of collection, sampling site, tissue choice and analytical technique may also influence the results.

Despite the methodological difficulties in assessing diet from the remote past, our FFQ for recent intake proves useful in categorising women by relative intakes, and can be used as a methodological tool in studies relating maternal diet during pregnancy and infant outcomes.

\section{Acknowledgements}

The authors wish to thank Dr Mauricio Henández Avila, Director of the Center for Population Health Research, for providing us with the topic for this study and also Dr Johanna Dwyer for supporting laboratory work at HNRC at Tufts University. Financial support was given by the Pan American Health Organization (PAHO)/World Health Organization/IDRC Grant MEX96/002721 GRT 'F', the 
Mexican National Research Council Grant 29115-M/2000, PAHO Grant No. Mex. 93-932 and the National Center for Environmental Health, Centers for Disease Control and Prevention (CDC), Atlanta, GA, USA.

\section{References}

1 British Nutrition Foundation. n-3 Fatty Acids and Health. Briefing Paper. London: BNF Publications (http://www. nutrition.org.uk), 2000.

2 Al MDM, van Houwelingen AC, Hornstra G. Long-chain polyunsaturated fatty acids, pregnancy, and pregnancy outcome. Am. J. Clin. Nutr. 2000; 71(Suppl.): 285S-91S.

3 Shaw CA, McEachern JC. The effects of early diet on synaptic function and behaviour: pitfalls and potentials. In: Dobbing J, ed. Developing Brain and Behaviour: The Role of Lipids in Infant Formula. London: Academic Press Limited, 1997; 427-74.

4 Farquharson J, Cockburn F, Patrick WA, Jamieson EC, Logan RW. Infant cerebral cortex phospholipid fatty-acid composition and diet. Lancet 1992; 340: 810-3

5 Gibson RA, Makrides M. n-3 Polyunsaturated fatty acid requirements of term infants. Am. J. Clin. Nutr. 2000; 71(Suppl.): 251S-5S.

6 Dutta-Roy AK. Transport mechanisms for long-chain polyunsaturated fatty acids in the human placenta. Am. J. Clin. Nutr. 2000; 71(Suppl.): 315S-22S.

7 Willett W. Nutritional Epidemiology. 2nd ed. Monographs in Epidemiology and Biostatistics. New York: Oxford University Press, 1998; 101-47, 174-243.

8 Block G, Hartman AM, Naughton D. A reduced dietary questionnaire: development and validation. Epidemiology 1990; 1: 58-64

9 Willett WC, Stampfer MJ, Underwood BA, Speizer FE, Rosner $\mathrm{B}$, Hennekens $\mathrm{CH}$. Validation of a dietary questionnaire with plasma carotenoid and alpha-tocopherol levels. Am.J. Clin. Nutr. 1983; 38: 631-9.

10 Romieu I, Parra-Cabrera S, Hernández-Avila JF, Madrigal H, Willett W, Hernández-Avila M. Questionnaire assessment of antioxidants and retinol intakes in Mexican women. Arch. Med. Res. 1999; 30: 224-39.

11 Bjerve KS, Brubakk AM, Fougner KJ, Johnsen H, Midthjell K, Vik T. Omega-3 fatty acids: essential fatty acids with important biological effects, and serum phospholipid fatty acids as markers of dietary $\omega 3$-fatty acid intake. Am.J. Clin. Nutr. 1993; 57(Suppl.): 801S-5S.

12 Popp-Snijders C, Schouten JA, van Blitterswijk WJ, van der Veen EA. Changes in membrane lipid composition of human erythrocytes after dietary supplementation of $(n-3)$ polyunsaturated fatty acids. Maintenance of membrane fluidity. Biochim. Biophys. Acta 1989; 854: 31-7.
13 von Shacky C, Fisher S, Weber PC. Long-term effects of dietary marine omega-3 fatty acids upon plasma and cellular lipids, platelet function, and eicosanoid formation in humans. J. Clin. Invest. 1985; 76: 1626-31.

14 Olsen SF, Hansen HS, Sandstrom B, Jensen B. Erythrocyte levels compared with reported dietary intake of marine $n-3$ fatty acids in pregnant women. Br. J. Nutr. 1995; 73: 387-95.

15 Harris M, Reece MS, McGregor JA, Manchego JM, Allen KDG. Possible roles of maternal and perinatal long-chain fatty acids in preterm birth. In: Huang Y-S, Sinclair A, eds. Lipids in Infant Nutrition. Champaign, IL: AOCS Press, 1998; 1-18.

16 Uauy-Dagach R, Mena P. Nutritional role of omega-3 fatty acids during the perinatal period. Clin. Perinatol. 1995; 22: $157-75$.

17 Olsen SF, Sorensen JD, Secher NJ, Hedegaard M, Henriksen TB, Hansen HS, et al. Randomised controlled trial of effect of fish-oil supplementation on pregnancy duration. Lancet 1992; 339: 1003-7.

18 Hernández-Avila M, Romieu I, Parra-Cabrera S, HernándezAvila JE, Madrigal H, Willett W. Validity and reproducibility of a food frequency questionnaire to assess dietary intake of women living in Mexico City. Salud Publica Mex. 1998; 40: $122-40$.

19 US Department of Agriculture, Agricultural Research Service. USDA Nutrient Database for Standard Reference, Release 14 [online]. Available at Nutrient Data Laboratory Home Page, http://www.nal.usda.gov/fnic/foodcomp, 2001.

20 US National Research Council, Subcommittee on the Tenth Edition of the RDAs. Recommended Dietary Allowances, 10th ed. Washington, DC: National Academy Press, 1989.

21 Hulshof KFAM, van Erp-Baart MA, Anttolainen M, Becker W, Church SM, Couet $\mathrm{C}$, et al. Intake of fatty acids in western Europe with emphasis on trans fatty acids: the TRANSFAIR Study. Eur. J. Clin. Nutr. 1999; 53: 143-57.

22 Hornstra G, Al MDM, van Houwelingen AC, Foreman-van Drongelen MM. Essential fatty acids in pregnancy and early human development. Eur. J. Obst. Gynecol. Reprod. Biol. 1995; 61: 57-62.

23 Araya AJ, Rojas GM, Fernández FP, Mateluna AA. Diferencias en la composición porcentual de los poliinsaturados de cadena larga en eritrocitos materno-fetales en nacimientos de término o pretérmino en humanos [Differences in percent composition of long chain polyunsaturated fatty acids in maternal-fetal erythrocytes in term and preterm infants]. Arch. Latinoam. Nutr. 1998; 48: 210-5.

24 von Houwelingen AC, Kester AD, Kromhout D, Hornstra G. Comparison between habitual intake of polyunsaturated fatty acids and their concentrations in serum lipid fractions. Eur. J. Clin. Nutr. 1989; 43: 11-20.

25 Arab L, Akbar J. Biomarkers and the measurement of fatty acids. Public Health Nutr. 2002; 5: 865-71. 